\title{
EFECTOS DEL CAMBIO CLIMÁTICO EN LA PRODUCCIÓN Y RENDIMIENTO DE DOS CULTIVOS AGRÍCOLAS EN PIURA, PERÚ 1973-2018
}

\author{
Villegas Yarleque, Mario ${ }^{1}$, Hidalgo Armestar, Ronald ${ }^{2}$, Villegas Aguilar, Gretel Fiorella ${ }^{3}$ \\ ORCID $^{3}$ : https//orcid.org/0000-0001-8145-1003 \\ Universidad Nacional de Frontera ${ }^{1}$ Sullana-Perú \\ Universidad Nacional de Frontera ${ }^{2}$ Sullana-Perú \\ Universidad Privada del Norte ${ }^{3} \quad$ Lima-Perú
} mvillegas@unf.edu.pe, ronaldhidalgoarmestar@gmail.com,grtlvillegasaguilar@gmail.com ORCID $^{1}$ :https://orcid.org/0000-0001-5572-1372 ORCID ${ }^{\wedge 2}$ : https://orcid.org/0000-0001-6502-3922

Recibido (05/10/20), Aceptado (20/10/20)

\begin{abstract}
Resumen: El calentamiento global ha alterado el cambio climático; por ello se investigó el comportamiento de la temperatura mínima, temperatura máxima, precipitación pluvial y su impacto en los cultivos de limón y maíz amarrillo duro. Para realizar la investigación, se utilizó como metodología la Función de producción y como parte de los resultados se calculó la primera derivada parcial para encontrar el umbral óptimo y la segunda derivada para encontrar su comportamiento convexo o cóncavo. Para el caso del limón se encontró que la Precipitación Pluvial y la Temperatura Máxima tuvieron un impacto negativo en la producción y rendimiento del limón, todo esto sucedió en el corto plazo ya que a largo plazo fue lo contrario. Para el caso del maíz amarrillo duro cuando la temperatura mínima sobrepasa el umbral la producción y rendimiento disminuye en cambio cuando la precipitación pluvial sobrepasa el umbral ésta aumenta.
\end{abstract}

Palabras Clave: Cambio climático, Función de producción agrícola, Umbrales óptimos, Variables climáticas

\section{EFFECTS OF CLIMATE CHANGE ON THE PRODUCTION AND YIELD OF TWO AGRICULTURAL CROPS IN PIURA, PERU 1973-2018}

\begin{abstract}
Global warming has altered climate change; For this reason, the behavior of the minimum temperature, maximum temperature, rainfall and their impact on lemon and hard yellow corn crops was investigated. To carry out the research, the Production Function was used as a methodology and as part of the results, the first partial derivative was calculated to find the optimal threshold and the second derivative to find its convex or concave behavior. In the case of lemon, it was found that Rainfall and Maximum Temperature had a negative impact on the production and yield of lemon, all this happened in the short term since in the long term it was the opposite. In the case of hard yellow corn, when the minimum temperature exceeds the threshold, production and yield decrease, on the other hand, when rainfall exceeds the threshold, it increases.
\end{abstract}

Keywords: Climate change, Agricultural production function, Optimal thresholds, Climatic variables. 


\section{I.INTRODUCCIÓN}

El impacto del cambio climático ha tenido gran repercusión en la agricultura en las últimas décadas alterando el cambio climático en todo el mundo. Según el Instituto Internacional de Investigacion sobre Politicas Alimentarias [1] detalló que el aumento irrestricto de gases esta subiendo la temperatura; donde la agricultura es el principal afectado ya que es vulnerable a estos cambios bruscos en el clima donde termina por perjudicar a la producción de cultivos y a la vez provoca las plagas y el crecimiento de hierbas malas, todo esto afectara negativamente a la agricultura y por ende al bienestar humano.

Mora [2] en su estudio titulado "Panama: Efectos del Cambio Climático sobre la agricultura" demostraron que a mayores niveles de precipitacion acumulada en los meses de mayo a octubre originaron beneficios en el cultivo ya que obtuvieron los mas altos índices de producción, también señalaron que de experimentar disminuciones en la precipitación acumulada los efectos serian contrarios lo que quiere decir que la producción de los cultivos reducirían. A nivel de cultivos para el maíz, el arroz y el banano la temperatura óptima permitiría generar mayores rendimientos en la producción y donde se siga teniendo esos incrementos en la temperatura los cultivos van a sufrir caidas considerables.

De acuerdo con Falco [3], en un estudio titulado "Estimación del impacto del cambio climático en la agricultura en países de bajos ingresos: evidencia a nivel de hogares de la cuenca del Nilo, Etiopía, utilizaron la productividad agrícola en un marco ricardiano, usando el método de interpolación espacial para predecir los valores específicos de la lluvia, encontraron que el cambio climático tiene un impacto significativo, tanto en los ingresos agricolas como la productividad agrícola.

Según Carrasco [4], en una investigación de la quinua en el Distrito de Juli, Puno, encontró que al elevarse $1{ }^{\circ} \mathrm{C}$ la temperatura máxima, la producción se reduce 112.2 TM. y el rendimiento $169.1 \mathrm{Kg} / \mathrm{Ha}$; por la elevación de las precipitaciones pluviales en $300 \mathrm{~mm}$ la producción se reduce en $75.78 \mathrm{TM}$ y el rendimiento en $127 \mathrm{Kg} / \mathrm{Ha}$.

Torres [5], en un estudio para la economía piurana de cultivos para la exportación como el mango, limón, café y plátano arrojó que el rendimiento de estos productos son explicados en mas de 50\% por variables climáticas, en base a las estimaciones realizadas se cuantificó pérdidas en el rendimiento de los cultivos en la proyección del año 2030, pero en el caso del café un mayor aumento de temperatura beneficia su producción y por ende el rendimiento hasta en un $41 \%$.

Para llevar a cabo la investigación, se utilizó una serie temporal y como metodología se usó la Función de producción agrícola, bajo el enfoque estructural, con el método de mínimos cuadrados ordinarios utilizando el criterio de Akaike y Schwarz para lograr estimar las principales variables como la temperatura mímima, la temperatura máxima y la precipitación pluvial que afectan a los cultivos del limón y del maíz amarillo duro de la región Piura en la producción y el rendimiento medido en $\mathrm{kg} / \mathrm{Hap}$, luego sacando la primera derivada parcial se encontró el umbral óptimo y calculando la segunda derivada se encontró su comportamiento ya sea convexo o cóncavo.

Para el caso del limón se encontró que la Precipitación Pluvial y la Temperatura Máxima tuvieron un impacto negativo en la producción del limón. En tanto para el rendimiento del limón este disminuyó cuando aumentó la precipitación pluvial y también cuando incrementó la temperatura máxima, todo esto sucedió en el corto plazo ya que a largo plazo fue lo contrario.

Para el caso del maíz amarrillo duro cuando la temperatura mínima sobrepasa el umbral la producción disminuye en cambio cuando la precipitación pluvial sobrepasa el umbral esta aumenta. En tanto las mismas variables influyen en el rendimiento de dicho producto.

\section{II.DESARROLLO}

Función de producción agrícola

La función de producción agrícola (Q) siguiendo a Fleischer [6] se expresó con base en las variables endogenas (x) que incluyen trabajo, fertilizantes, capital $\mathrm{y}$ otros insumos. Las variables exogenas (z) expresan a las variables climaticas y las caracteristicas de los agricultores (m).

Por tanto, la funcion de producción agrícola se presenta en la siguiente ecuación:

$$
Q_{t}=f\left(m_{t}, z_{t}, x_{t}\right)
$$

Donde Qt representa la producción agropecuaria y el sub índice $t$ indica el tiempo considerado en el estudio

Asi la función de beneficios de un agricultor que produce $n$ cultivos en un tiempo determinado $t$ se expresa de la siguiente forma:

$$
\pi_{t}=\sum_{j=1}^{1}\left[p_{j t} \mathrm{Q}_{j t}\left(m_{t}, z_{t}, x_{j t}\right)-w_{t} x_{j t}\right] \mathrm{j}=1,2 \ldots . \mathrm{n} \text { cultivos }
$$

Donde pj representa los precios del producto $\mathrm{j} \mathrm{y} \mathrm{w}$ los precios de los insumos del producto $\mathrm{j}$

Los agricultores en este enfoque de la producción buscan maximizar sus beneficios y por lo que eligen 
una cierta cantidad de insumos (x) considerando las variables exógenas $(\mathrm{z})$ como el clima.

La cantidad óptima de los insumos seleccionados debe satisfacer la siguiente condición de primer orden en cada uno de los periodos considerados en el estudio:

$$
p j \frac{\partial \mathrm{Q}_{t}}{\partial x_{t}}=w \quad \mathrm{w}, \quad \mathrm{j}=1,2, \ldots \mathrm{n}
$$

En esta investigación, y partiendo de los enfoques de producción antes expuestos se evaluará las variables climáticas consideradas como exógenas y su impacto en la producción de los cultivos más importantes de la región Piura. La siembra de los cultivos se desarrolla dependiendo de la humedad y temperatura, durante su etapa de crecimiento.

\section{III.METODOLOGÍA}

En ésta investigación la metodología que se utilizó para estimar el rendimiento de cada cultivo propuesto en función a las variables independientes que fueron la superficie cosechada, los cambios climáticos donde se consideró la temperatura mínima, la temperatura máxima y las precipitaciones pluviales, se realizó una comparación con cada una de las temperaturas para ver el efecto individual de cada variable, a continuación se estimó las variables en conjunto tanto de temperatura mínima y temperatura máxima, para luego estimar las precipitaciones pluviales. La secuencia de estas estimaciones nos permitirá analizar el efecto de cada variable para luego comparar y elegir el mejor modelo mediante los criterios econométricos y estadísticos.

El área de estudio. Según el Banco Central de Reserva del Perú [7], el departamento de Piura se ubica al noroeste del país, tiene una extensión de $35892 \mathrm{~km} 2$, que ocupa el 3,1\% del territorio nacional, políticamente se conforma por 8 provincias y 65 distritos, siendo su capital la ciudad de Piura. Posee un clima tropical y seco con una temperatura anual de $24^{\circ} \mathrm{C}$ y en verano supera los $35^{\circ} \mathrm{C}$, las épocas de lluvia es en enero y marzo. En las zonas andinas el clima presenta noches frías y templadas. El sector agropecuario en el periodo 2008-2017 registro un crecimiento promedio anual de 3.3\%. En el año 2017 por el fenómeno del niño costero se perdieron miles de hectáreas donde se dañó la infraestructura productiva y se interrumpió la actividad comercial.

Datos. Como información para elaborar la base de datos se utilizó series de tiempo extraídas del Ministerio de agricultura y riego usando el sistema de series estadísticas de la producción agrícola (SEPA) y del Servicio Nacional de Meteorología e Hidrología (SENAMHI) para extraer las variables de temperatura máxima, temperatura mínima y precipitación pluvial.

El modelo. El modelo econométrico siguiendo el método de la función de producción polinómica de segundo grado para el análisis de rendimiento y producción es el siguiente:

$$
\begin{gathered}
R_{a t}=\beta_{0}+\beta_{1} \operatorname{Tmin}_{a t}+\beta_{2}\left(\operatorname{Tmin}_{a t}\right)^{2}+\beta_{3} \operatorname{Tmax}_{a t}+\beta_{4}\left(\operatorname{Tmax}_{a t}\right)^{2} \\
+\beta_{5} \operatorname{Precip}_{a t}+\beta_{6}\left(\text { Precip }_{a t}\right)^{2}+\mu_{t} \\
Q_{a t}=\beta_{0}+\beta_{1} S_{a t}+\beta_{2} \text { Tmin }_{a t}+\beta_{3}\left(\operatorname{Tmin}_{a t}\right)^{2}+\beta_{4} \operatorname{Tmax}_{a t} \\
+\beta_{5}\left(\operatorname{Tmax}_{a t}\right)^{2}+\beta_{6} \text { Precip }_{a t}+\beta_{7}\left(\text { Precip }_{a t}\right)^{2}+\mu_{t}
\end{gathered}
$$

Para encontrar los niveles óptimos de temperatura, se empleó la primera derivada para sacar el valor óptimo de las variables climáticas y la segunda derivada para ver si tiene relación convexa o cóncava.

\section{IV.RESULTADOS}

Producción de limón: Analizando los parámetros empleando MCO se encontró que la precipitación pluvial y la temperatura máxima tienen un impacto negativo en la producción del limón. Analizando el R2 hay una correlación fuerte y positiva debido a que las variables independientes explican en un $77 \%$ a la variable dependiente. 
Tabla I. Producción de limón

\begin{tabular}{|c|c|c|c|c|c|c|}
\hline VARIABLES & Modelo 1 & Modelo 2 & Modelo 3 & Modelo 4 & Modelo 5 & Modelo 6 \\
\hline \multirow[t]{2}{*}{ S_LIMON } & $10.81^{* * *}$ & $10.92 * * *$ & $10.93^{* * *}$ & $10.79^{* * *}$ & $10.62^{* * *}$ & $9.860^{* * *}$ \\
\hline & $(1.250)$ & $(1.180)$ & $(1.199)$ & $(1.215)$ & $(1.059)$ & (1.016) \\
\hline \multirow[t]{2}{*}{ Tmin } & $-41,980$ & $-150,711$ & $-219,090$ & $-205,681$ & & \\
\hline & $(287,767)$ & $(161,241)$ & $(155,032)$ & $(155,370)$ & & \\
\hline \multirow[t]{2}{*}{$\operatorname{Tmin} 2$} & 1,011 & 3,536 & 5,649 & 5,144 & & \\
\hline & $(7,415)$ & $(4,035)$ & $(3,908)$ & $(3,880)$ & & \\
\hline \multirow[t]{2}{*}{ Tmax } & $-499,443$ & $-83,764$ & & & $-562,270$ & $-202,519$ \\
\hline & $(726,434)$ & $(264,067)$ & & & $(393,478)$ & $(249,104)$ \\
\hline \multirow[t]{2}{*}{$\operatorname{Tmax} 2$} & 8,193 & 1,598 & & & 9,186 & 3,377 \\
\hline & $(11,829)$ & $(4,200)$ & & & $(6,344)$ & $(3,964)$ \\
\hline \multirow[t]{2}{*}{ PRECIP } & $-101.9^{* *}$ & & $-102.4^{* *}$ & & $-107.8^{* *}$ & \\
\hline & $(47.93)$ & & $(43.57)$ & & $(42.85)$ & \\
\hline PRECIP2 & $0.0417^{*}$ & & $0.0357^{*}$ & & $0.0441^{* *}$ & \\
\hline \multirow[t]{2}{*}{ Constant } & $8.050 \mathrm{e}+06$ & $2.654 \mathrm{e}+06$ & $2.129 \mathrm{e}+06$ & $2.051 \mathrm{e}+06$ & $8.612 \mathrm{e}+06$ & $3.042 \mathrm{e}+06$ \\
\hline & $(9.022 \mathrm{e}+06)$ & $(3.874 \mathrm{e}+06)$ & $(1.533 \mathrm{e}+06)$ & $(1.547 \mathrm{e}+06)$ & $(6.101 \mathrm{e}+06)$ & $(3.911 \mathrm{e}+06)$ \\
\hline $\mathrm{N}$ & 43 & 46 & 43 & 46 & 43 & 46 \\
\hline $\mathrm{R}^{2}$ & 0.775 & 0.744 & 0.771 & 0.710 & 0.774 & 0.723 \\
\hline $\mathrm{R}^{2}$ ajustado & 0.73 & 0.71 & 0.74 & 0.69 & 0.74 & 0.70 \\
\hline $\mathrm{AK}$ & 1008.00 & 1077.67 & 1004.72 & 1079.36 & 1004.13 & 1077.18 \\
\hline $\mathrm{SCH}$ & 1022.09 & 1088.64 & 1015.29 & 1086.68 & 1014.69 & 1084.49 \\
\hline
\end{tabular}

Standard errors in parentheses

$$
* * * \mathrm{p}<0.01, * * \mathrm{p}<0.05, * \mathrm{p}<0.1
$$

Rendimiento del Limón : En tanto para el ren- con las lineales, el coeficiente de correlación es muy dimiento del limón éste disminuye cuando aumenta la bajo y positivo debido a que las variables independienprecipitación pluvial y también cuando incrementa la tes explican en un $18 \%$ a la variable dependiente, esto temperatura máxima (de la misma manera que en el genera presencia de multicolinealidad que nos arroja resultado anterior), en tanto en el modelo estimado no ineficiencia en las variables cuadráticas pero no las elihay significancia estadística ya que se ve afectado por minamos para poder encontrar los efectos no lineales. las variables cuadráticas que están altamente relacionas

Tabla II. Rendimiento de limón

\begin{tabular}{lcccccc}
\hline VARIABLES & Modelo 1 & Modelo 2 & Modelo 3 & Modelo 4 & Modelo 5 & Modelo 6 \\
\hline \multirow{2}{*}{ Tmin } & & & & & & \\
& $-2,084$ & -940.7 & $-16,037$ & $-10,448$ & & \\
Tmin2 & $(27,196)$ & $(14,176)$ & $(14,697)$ & $(13,805)$ & & \\
& 43.15 & -2.209 & 408.1 & 256.7 & & \\
Tmax & $(703.7)$ & $(357.0)$ & $(372.3)$ & $(346.6)$ & & \\
& $-41,593$ & $-27,090$ & & & $-42,233$ & $-32,167$ \\
Tmax2 & $(70,284)$ & $(25,161)$ & & & $(38,841)$ & $(23,949)$ \\
& 680.5 & 456.0 & & & 685.4 & 522.2 \\
PRECIP & $(1,145)$ & $(400.3)$ & & & $(626.2)$ & $(381.2)$ \\
& -3.553 & & -3.353 & & -4.725 & \\
PRECIP2 & $(4.686)$ & & $(4.198)$ & & $(3.912)$ & \\
& 0.000731 & & 0.000142 & & 0.00112 & \\
Constant & $(0.00235)$ & & $(0.00199)$ & & $(0.00193)$ & \\
& 670,625 & 431,621 & 168,438 & 116,734 & 661,603 & 505,929 \\
& $(879,359)$ & $(371,339)$ & $(144,868)$ & $(137,173)$ & $(602,203)$ & $(375,974)$ \\
\hline N & & & & & & \\
R2 & 43 & 46 & 43 & 46 & 43 & 46 \\
R2 ajustado & 0.189 & 0.148 & 0.180 & 0.020 & 0.183 & 0.073 \\
AK & 0.05 & 0.06 & 0.09 & -0.03 & 0.10 & 0.03 \\
SCH & 807.85 & 861.15 & 804.31 & 863.60 & 804.14 & 861.01 \\
& 820.18 & 870.29 & 813.11 & 869.09 & 812.94 & 866.50 \\
\hline
\end{tabular}

Standard errors in parentheses

$* * * \mathrm{p}<0.01, * * \mathrm{p}<0.05, * \mathrm{p}<0.1$ 
En un estudio elaborado por Lázaro [8], muestra que la temperatura es un factor limitante para el cultivo donde la temperaturas favorables oscilan entre $25^{\circ}$ la mínima y la máxima $30^{\circ} \mathrm{C}$, en tanto la precipitacion pluvial es preferible que sea de 400 a $1200 \mathrm{~mm}$ anuales. Calculando las derivadas parciales de las variables que más impacto tienen en la producción de limón se presentan los siguientes resultados:

Producción $Q L=8612+1062$ Slimon -107.8 precip + 0.0441 precip $^{2}-562270$ tmax +9186 tmax ${ }^{2}$

Tabla III. Derivadas de la producción de Limón

\begin{tabular}{|l|r|r|r|}
\hline Variables & Primera derivada & Segunda derivada & Unidad de medida \\
\hline Tmax & 30.605 & $18372>0$ & ${ }^{\circ} \mathrm{C}$ \\
\hline Precip & 1222.22 & $0.0882>0$ & $\mathrm{~mm}$ \\
\hline
\end{tabular}

Producción y rendimiento del maíz amarillo duro

En la tabla 4 y 5 se consideró como mejor modelo el 3 para ambas estimaciones ya que ese modelo tiene una mayor robustez y el menor $\mathrm{AK}$ y SCH, analizando estos parámetros dio como resultado que la temperatura mínima influye positivamente en la producción de maíz mientras que la precipitación pluvial afecta a la produc- ción, en tanto hay una correlación fuerte y positiva lo que indica que las variables independientes explican en un $93 \%$ a la variable dependiente.

Para el rendimiento de $\mathrm{Kg}$ por hectárea, se dieron los mismos resultados que en la producción pero con diferentes parámetros, la temperatura mínima es significativa al $95 \%$ y la precipitación es no significativa debido a que influye en el modelo en niveles bajos, en tanto hay una correlación baja y positiva que nos dice que las variables independientes explican en un $27 \%$ a la dependiente esto indica que hay presencia de multicolinealidad, ya que las estimaciones lineales son similares con las cuadráticas y esto genera que una de las dos variables sea irrelevante, ya que no está aportando información adicional con respecto a la otra, pero como se mencionó anteriormente se mantuvieron las variables cuadráticas con la finalidad de encontrar los efectos no lineales.

Calculando las derivas parciales de la mejor estimación de la producción se obtuvieron los siguientes resultados:

$$
\begin{gathered}
Q \operatorname{mad}=-440037+3.662 \text { Smad }-14.17 \text { precip }+ \\
0.00578 \text { precip }^{2}+42458 \text { tmin }-1019 \text { tmin }^{2}
\end{gathered}
$$

\begin{tabular}{|c|c|c|c|c|c|c|}
\hline VARIABLES & Modelo 1 & Modelo 2 & Modelo 3 & Modelo 4 & Modelo 5 & Modelo 6 \\
\hline S_MAIZ & $\begin{array}{c}3.617 * * * \\
(0.256)\end{array}$ & $\begin{array}{c}3.604 * * * \\
(0.234)\end{array}$ & $\begin{array}{c}3.662 * * * \\
(0.243)\end{array}$ & $\begin{array}{c}3.579 * * * \\
(0.220)\end{array}$ & $\begin{array}{c}3.881 * * * \\
(0.215)\end{array}$ & $\begin{array}{c}3.778 * * * \\
(0.174)\end{array}$ \\
\hline Tmin & $\begin{array}{c}37,648 \\
(62,819)\end{array}$ & $\begin{array}{c}33,812 \\
(39,687)\end{array}$ & $\begin{array}{c}42,458 \\
(38,471)\end{array}$ & $\begin{array}{c}45,667 \\
(35,293)\end{array}$ & & \\
\hline Tmin 2 & $\begin{array}{l}-867.1 \\
(1,616)\end{array}$ & $\begin{array}{l}-815.8 \\
(991.6)\end{array}$ & $\begin{array}{l}-1,019 \\
(969.9)\end{array}$ & $\begin{array}{l}-1,123 \\
(882.0)\end{array}$ & & \\
\hline Tmax & $\begin{array}{c}4,842 \\
(153,438)\end{array}$ & $\begin{array}{c}36,709 \\
(57,421)\end{array}$ & & & $\begin{array}{c}15,169 \\
(92,380)\end{array}$ & $\begin{array}{c}62,336 \\
(51,360)\end{array}$ \\
\hline Tmax2 & $\begin{array}{l}-107.2 \\
(2,499)\end{array}$ & $\begin{array}{l}-598.0 \\
(912.7)\end{array}$ & & & $\begin{array}{l}-226.0 \\
(1,490)\end{array}$ & $\begin{array}{l}-990.1 \\
(817.4)\end{array}$ \\
\hline PRECIP & $\begin{array}{l}-16.18 \\
(10.56)\end{array}$ & & $\begin{array}{l}-14.17 \\
(9.789)\end{array}$ & & $\begin{array}{l}-10.50 \\
(10.34)\end{array}$ & \\
\hline PRECIP2 & $\begin{array}{c}0.00600 \\
(0.00538)\end{array}$ & & $\begin{array}{c}0.00578 \\
(0.00469)\end{array}$ & & $\begin{array}{c}0.00481 \\
(0.00518)\end{array}$ & \\
\hline Constant & $\begin{array}{c}-450,900 \\
(1.926 \mathrm{e}+06)\end{array}$ & $\begin{array}{l}-912,796 \\
(815,183)\end{array}$ & $\begin{array}{l}-440,037 \\
(379,147)\end{array}$ & $\begin{array}{l}-463,394 \\
(350,398)\end{array}$ & $\begin{array}{c}-256,816 \\
(1.431 \mathrm{e}+06)\end{array}$ & $\begin{array}{c}-983,854 \\
(806,271)\end{array}$ \\
\hline $\mathrm{N}$ & 43 & 46 & 43 & 46 & 43 & 46 \\
\hline $\mathrm{R}^{2}$ & 0.930 & 0.922 & 0.929 & 0.921 & 0.923 & 0.919 \\
\hline $\mathrm{R}^{2}$ ajustado & 0.92 & 0.91 & 0.92 & 0.92 & 0.91 & 0.91 \\
\hline $\mathrm{AK}$ & 875.78 & 934.35 & 872.40 & 931.21 & 876.00 & 932.10 \\
\hline $\mathrm{SCH}$ & 889.87 & 945.32 & 882.96 & 938.53 & 886.57 & 939.42 \\
\hline
\end{tabular}

Tabla IV. Producción de maíz amarillo duro 
Tabla V. Rendimiento de maíz amarillo duro

\begin{tabular}{|c|c|c|c|c|c|c|}
\hline $\begin{array}{l}\text { VA RIABLE } \\
\mathrm{S}\end{array}$ & Modelo 1 & Modelo 2 & Modelo 3 & Modelo 4 & Modelo 5 & Modelo 6 \\
\hline Tmin & $\begin{array}{c}4,825 \\
(3,887)\end{array}$ & $\begin{array}{l}4,772^{* *} \\
(2,033)\end{array}$ & $\begin{array}{l}5,390^{* *} \\
(2,109)\end{array}$ & $\begin{array}{c}5,219^{* * *} \\
(1,856)\end{array}$ & & \\
\hline Tmin 2 & $\begin{array}{l}-114.9 \\
(100.6)\end{array}$ & $\begin{array}{c}-116.5^{* *} \\
(51.21)\end{array}$ & $\begin{array}{c}-131.3^{* *} \\
(53.43)\end{array}$ & $\begin{array}{c}-128.4 * * * \\
(46.61)\end{array}$ & & \\
\hline Tmax & $\begin{array}{c}112.5 \\
(10,046)\end{array}$ & $\begin{array}{c}1,560 \\
(3,609)\end{array}$ & & & $\begin{array}{c}6,841 \\
(6,333)\end{array}$ & $\begin{array}{c}5,092 \\
(3,634)\end{array}$ \\
\hline $\operatorname{Tmax} 2$ & $\begin{array}{l}-3.900 \\
(163.6)\end{array}$ & $\begin{array}{l}-25.66 \\
(57.41)\end{array}$ & & & $\begin{array}{l}-108.7 \\
(102.1)\end{array}$ & $\begin{array}{l}-80.30 \\
(57.83)\end{array}$ \\
\hline PRECIP & $\begin{array}{l}-0.882 \\
(0.670)\end{array}$ & & $\begin{array}{l}-0.686 \\
(0.602)\end{array}$ & & $\begin{array}{c}0.275 \\
(0.638)\end{array}$ & \\
\hline PRECIP2 & $\begin{array}{c}0.000295 \\
(0.000336)\end{array}$ & & $\begin{array}{c}0.000251 \\
(0.000286)\end{array}$ & & $\begin{array}{l}-0.000183 \\
(0.000314)\end{array}$ & \\
\hline Constant & $\begin{array}{c}-46,519 \\
(125,693)\end{array}$ & $\begin{array}{l}-68,903 \\
(53,261)\end{array}$ & $\begin{array}{c}-51,545^{* *} \\
(20,789)\end{array}$ & $\begin{array}{c}-49,396^{* *} \\
(18,445)\end{array}$ & $\begin{array}{l}-104,082 \\
(98,183)\end{array}$ & $\begin{array}{l}-77,169 \\
(57,045)\end{array}$ \\
\hline $\mathrm{N}$ & 43 & 46 & 43 & 46 & 43 & 46 \\
\hline $\mathrm{R}^{2}$ & 0.280 & 0.220 & 0.266 & 0.211 & 0.057 & 0.051 \\
\hline $\mathrm{R}^{2}$ ajustado & 0.16 & 0.14 & 0.19 & 0.17 & -0.04 & 0.01 \\
\hline AK & 640.55 & 682.49 & 637.35 & 679.01 & 648.15 & 687.53 \\
\hline $\mathrm{SCH}$ & 652.88 & 691.63 & 646.15 & 684.50 & 656.96 & 693.02 \\
\hline
\end{tabular}

En un estudio elaborado por Yzarra, [9]especificó que el maiz en tierras altas crece y se desarrolla en temperaturas más bajas que a los cultivos adaptados a las tierras bajas, para ello la temperatura óptima en tierras bajas se adopta entre $30^{\circ}$ y $34^{\circ} \mathrm{C}$ y se considera que para el maíz tropical de tierras altas esta alrededor de $21^{\circ} \mathrm{C}$.

Tabla VI. derivadas de la producción de Maíz Amarrillo Duro

\begin{tabular}{|l|r|r|r|}
\hline Variables & Primera derivada & Segunda derivada & Unidad de medida \\
\hline Tmin & 20.83 & $2038>0$ & ${ }^{\circ} \mathrm{C}$ \\
\hline Precip & 1225.77 & $0.01156>0$ & $\mathrm{~mm}$ \\
\hline
\end{tabular}

\section{V.CONCLUSIONES}

Los resultados de los análisis arrojaron que las variables climáticas y la precipitación tienen efectos tanto en la producción como en los rendimientos de los 2 cultivos en la región Piura y a éstos modelos los avalaron la bondad de ajustes, ya que hay una explicación entre las variables incluso en más de $50 \%$.

1) Para el caso de la producción y rendimiento del limón, las variables que más impacto tiene en el cultivo es la temperatura máxima y la precipitación plu- vial; para la temperatura máxima a medida que se eleva sobrepasando del umbral óptimo que es de $30.605^{\circ} \mathrm{C}$ la producción aumenta, es decir al elevarse en $1^{\circ} \mathrm{C}$ la temperatura máxima la producción aumenta en 9186 toneladas de limón, en tanto para la precipitación pluvial si sobrepasa el umbral óptimo que es de $1222.22 \mathrm{~mm}$ se incrementa la producción, es decir si la precipitación pluvial se incrementa en $1 \mathrm{~mm}$ la producción de limón aumenta en 0.0441 toneladas. En el rendimiento de limón, son las mismas variables climáticas que tienen impacto en el cultivo llegando a la conclusión que la temperatura máxima una vez alcanzado su umbral óptimo que es de $30.81^{\circ} \mathrm{C}$ el rendimiento aumenta, es decir si la temperatura máxima incrementa en $1{ }^{\circ} \mathrm{C}$ el rendimiento de limón tiene una tendencia creciente en 685.4 $\mathrm{Kg}$ /hectáreas, para la precipitación su umbral óptimo es de $2109.375 \mathrm{~mm}$, es decir si la precipitación pluvial se incrementa en $1 \mathrm{~mm}$ el rendimiento de limón se incrementa en $0.00112 \mathrm{Kg} /$ hectáreas, esto se explica ya que la producción de limón como el rendimiento tiene una relación cóncava, lo que indica que tiene la forma de una $\mathrm{U}$ invertida

2) Para el caso de la producción y rendimiento del maíz amarillo duro, las variables que más impacto tiene 
en el cultivo es la temperatura mínima y la precipitación pluvial, para la temperatura mínima a medida que sobrepasa el umbral óptimo que es de $20.83{ }^{\circ} \mathrm{C}$ la producción disminuye, es decir al disminuir $1^{\circ} \mathrm{C}$ la temperatura mínima se llega a la conclusión que la producción disminuye en 1019 toneladas de maíz amarillo duro, en tanto para la precipitación pluvial si sobrepasa el umbral óptimo que es de $1225.77 \mathrm{~mm}$ se incrementa la producción, es decir si la precipitación pluvial se incrementa en $1 \mathrm{~mm}$ la producción de maíz amarillo duro aumenta en 0.00578 toneladas. En el rendimiento de maíz amari1lo duro, son las mismas variables climáticas que tienen impacto en el cultivo llegando a la conclusión que la temperatura mínima una vez alcanzado su umbral óptimo que es de $20.525^{\circ} \mathrm{C}$ el rendimiento disminuye, es decir si la temperatura mínima se incrementa en $1^{\circ} \mathrm{C}$ el rendimiento de maíz tiene una tendencia decreciente de $131.3 \mathrm{Kg}$ /hectáreas, para la precipitación su umbral óptimo es de $1366.5338 \mathrm{~mm}$, es decir si la precipitación pluvial se incrementa en $1 \mathrm{~mm}$ el rendimiento de maíz amarillo duro se incrementa en $0.000251 \mathrm{Kg}$ /hectáreas.

3) Existen otros cultivos como la uva, el mango, el banano orgánico que son igualmente relevantes para la economía de los agricultores de la Región Piura cuyo destino es la exportación y que también se ven afectados por las mismas variables investigadas.

\section{REFERENCIAS}

[1]Instituto Internacional de Investigacion sobre Politicas Alimentarias, «Cambio Climatico,» Washington,

\section{RESUMEN CURRICULAR}

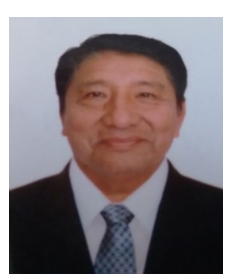

Mario Villegas Yarlequé, Economista con maestría en Ciencias de la Educación Superior, con experiencia en el sector privado en empresas de transporte interprovincial de pasajeros y sector público, en gobiernos locales y regionales; con 18 años de experiencia académica en universidades públicas y privadas. Investigador en la línea de Población y Desarrollo Sostenible. Actualmente me desempeño como docente auxiliar a tiempo completo en la Universidad Nacional de Frontera en la Facultad de Ingeniería Económica desde Marzo del 2017 hasta la actualidad, en calidad de docente nombrado.E-mail: mvillegas@unf.edu.pe

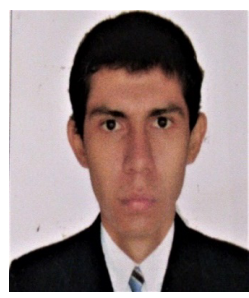

Ronald Hidalgo Armestar, Bachiller en ingeniería económica de la Universidad Nacional de Frontera, con experiencia como asistente de proyectos de investigación. Actualmente estudiante del curso de especialización en Econometría Aplicada de la Universidad Nacional de Ingeniería y dedicado a la producción científica.E-mail: ronaldhidalgoarmestar@gmail.com
D.C., 2009.

[2]J. Mora, D. Ramírez, J. Ordaz, A. Acosta y S. Braulio, «Panama Efectos del Cambio Climatico sobre la Agricultura,» Impreso en Naciones Unidas - México, D. F., Mexico, 2010.

[3]S. Falco, Y. Mahmud, G. Kohlin y C. Ringler, «Estimating the Impact of Climate Change on Agriculture in Low-Income Countries: Household Level Evidence from the Nile Basin, Ethiopia,» springer link, vol. 52, $n^{\circ} 457-478,2012$.

[4]F. Carrasco, «Efectos del cambio climático en la producción y rendimiento de la quinua en el distrito de Juli, periodo 1997 - 2014,»Comuni@cción, vol. 7, n² 2, p. 10, 2016.

[5]L. Torres, «Analisis Economico del Cambio Climatico en la Agricultura de Region Piura,» Consorcio de Investigación Económica y social, Piura, 2010.

[6]A. Fleischer, I. Lichtman y R. Mendelsohn, «Climate Change, Irrigation, and Israeli Agriculture: will warming be harmful ?,» No. 4135, Banco Mundial., 2007. [7]Banco Central de Reserva del Perú Sucursal Piura, «Caracterización deL departamento de Piura,» Piura .

[8]A. Lázaro, «Sustentabilidad de fincas productoras de Opuntia ficus indica para la producción de Dactylopius coccus,en Arequipa,» Lima, 2019.

[9]W. Yzarra, I. Trebejo y V. Noriega, «Evaluación del efecto del clima en la producción y productividad del maíz amarillo duro en la costa central del Perú,» Lima, 2010 .

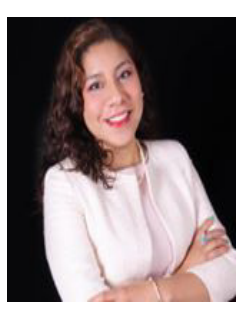

Gretel Fiorella Villegas Aguilar, Docente mentor a cargo de desarrollar nuevos modelos de negocios sostenibles con los estudiantes y ayudarlos en su formación académica profesional en Administración de Empresas en la Universidad Privada del Norte. Grado de Magister con mención en Docencia Universitaria e Investigación Pedagógica. Licenciada en Administración de empresas. Estudios de especialización en creación de nuevos modelos de negocios.E-mail: grtlvillegasaguilar@gmail.com 